\title{
A microscopic approach to heating rate of ferrofluid droplets by a magnetic field
}

Cite as: J. Appl. Phys. 125, 045104 (2019); https://doi.org/10.1063/1.5078648

Submitted: 26 October 2018 . Accepted: 08 January 2019. Published Online: 23 January 2019

E. C. Siqueira (D) L. R. N. Junior, A. R. Jurelo, J. F. H. L. Monteiro (D, P. A. Orellana, G. G. Cabrera, and F. F. Fachini (iD

\section{ARTICLES YOU MAY BE INTERESTED IN}

Neuromorphic thermal-electric circuits based on phase-change $\mathrm{VO}_{2}$ thin-film memristor elements

Journal of Applied Physics 125, 044501 (2019); https://doi.org/10.1063/1.5037990

The total secondary electron yield of a conductive random rough surface Journal of Applied Physics 125, 043301 (2019); https://doi.org/10.1063/1.5023769

Insight of the thermal conductivity of $\epsilon$-iron at Earth's core conditions from the newly developed direct ab initio methodology

Journal of Applied Physics 125, 045102 (2019); https://doi.org/10.1063/1.5055389

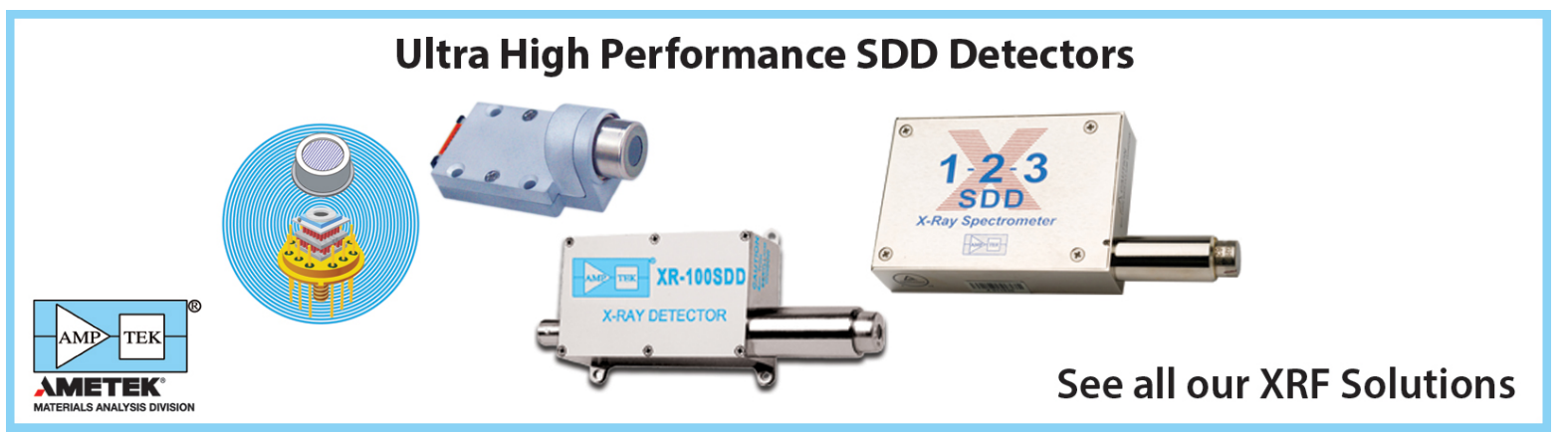




\title{
A microscopic approach to heating rate of ferrofluid droplets by a magnetic field
}

\author{
Cite as: J. Appl. Phys. 125, 045104 (2019); doi: 10.1063/1.5078648 \\ Submitted: 26 October 2018 - Accepted: 8 January 2019 . \\ Published Online: 23 January 2019
}

\author{
E. C. Siqueira, , a) (iD L. R. N. Junior, ${ }^{7}$ A. R. Jurelo, ${ }^{2}$ J. F. H. L. Monteiro, ${ }^{2}$ (D P. A. Orellana, ${ }^{3}$ G. G. Cabrera, ${ }^{4}$ \\ and F. F. Fachini ${ }^{5}$ (D)
}

\begin{abstract}
AFFILIATIONS
${ }^{1}$ Departamento de Física, Universidade Tecnológica Federal do Paraná, Ponta Grossa, Parana 84016-210, Brazil

${ }^{2}$ Departamento de Física, Universidade Estadual de Ponta Grossa, Ponta Grossa, Parana 84030-000, Brazil

${ }^{3}$ Departamento de Física, Universidad Técnica Federico Santa Maria, Av. Vicuña Mackenna 3939, Santiago, Chile

${ }^{4}$ Instituto de Física "Gleb Wataghin," Universidade Estadual de Campinas, Campinas, São Paulo 13083-859, Brazil

${ }^{5}$ Grupo de Mecânica de Fluidos Reativos, Instituto Nacional de Pesquisas Espaciais, Cachoeira Paulista, São Paulo 12630-000, Brazil
\end{abstract}

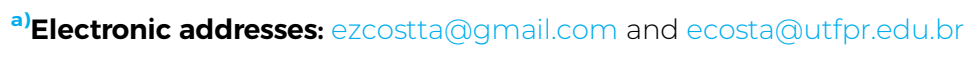

\begin{abstract}
In this work, we study the heating process of colloidal ferrofluids by a magnetic field. The heating of the fluid occurs by the magnetic relaxation of the nanoparticles which provide thermal energy for the host liquid. In the limit of small volumes, the relaxation process occurs through the Néel mechanism since the magnetic nanoparticles present superparamagnetic behavior. Within this limit, we have used a microscopic model for the coupling to phonons and external magnetic field in order to model the relaxation mechanism and to obtain an expression for the heating rate of the fluid as a function of microscopic parameters. The analysis allows determining appropriate conditions for an optimal heating rate for ferrofluids based on superparamagnetic nanoparticles.
\end{abstract}

Published under license by AIP Publishing. https://doi.org/10.1063/1.5078648

\section{INTRODUCTION}

Heating of colloidal ferrofluids is an emerging field due to its potential in technological applications. In fact, one of these applications is the so-called magnetic hyperthermia $(\mathrm{MH})$, where the ferrofluid is used to provide the heating of a system. ${ }^{1-11}$ When an external magnetic field is applied, induced magnetization lags the external field which results in heat dissipation at the fluid. Ferrofluids can be of interest in the construction of combustion chambers, whose projected size increases with the time taken for heating, vaporization, and consequently, burning of the fuel droplets. When magnetic nanoparticles are embedded in these droplets, it is possible to accelerate those processes allowing the construction of more compact chambers. ${ }^{12-14}$

Ferrofluids contain magnetic particles whose mobility can be controlled by a magnetic field. Regarding the applications in $\mathrm{MH}$, the interest is the absorption of large amounts of energy when the magnetization of the particles is reversed.
Thus, the quantity to be maximized is the heating rate, i.e., the amount of energy absorbed per unit of time. ${ }^{15-18}$ In the process of heating, the reversal of the magnetization can be accomplished by two ways: either the magnetic moment is rotated inside the particle until it is aligned with the field, which is called the Néel process, or the particle rotates as a whole, which is called the Brownian process. ${ }^{19-25}$ These mechanisms occur with a well defined relaxation time, i.e., the time that the particle takes to revert its magnetization. Besides the magnetization reversal, the heat transfer is different in these two processes. In the Brownian process, the heat is transferred directly to the fluid due to the friction generated by the particle rotation in the fluid. In the Néel process, the heat is first transferred to the lattice, i.e., to the phonons, and then is delivered to the fluid by heat transfer.

Several research studies have been done in the sense of achieving higher heating rates but considering a regime where both relaxation processes are operating at the same 
time. $^{12,25}$ However, in situations where the size of the particles is very reduced or the particles are immobilized, the Néel mechanism rules the thermal relaxation. In fact, the Néel relaxation time is reduced exponentially with the volume of the particle, while the Brownian mechanism is reduced linearly with volume. Accordingly, as an approximation, only the Néel mechanism is taken into account by assuming the limit of small volumes. ${ }^{26,27}$ In applications of ferrofluids in combustion, the magnetic nanoparticles must be embedded into the fuel droplets but avoiding significant changes of fluid properties. In this way, the size of the magnetic nanoparticles must be very reduced in order to achieve higher concentrations. In these cases, it is imperative to study the Néel contribution separately in order to have a deep understanding of the heating process. In this work, the Néel relaxation is considered as being a result of the coupling between the spin of the magnetic nanoparticle with the phonons of its crystalline lattice and an external magnetic field. To do this, a relaxation model of superparamagnetic nanoparticles, ${ }^{28}$ considering the spin-phonon interaction, is applied.

In this work, the magnetic hyperthermia is treated by using an approach based on quantum mechanics, in contrast with classical methods used to study magnetic hyperthermia. ${ }^{29-31}$ The theory of crystal field has been applied to model the Mössbauer spectra and other magnetic properties of materials, and here, we show that this theory may also be used in the study of applied technologies. In addition to magnetic hyperthermia, we have considered the application in spray combustion found in many devices. ${ }^{32-37}$ Since liquid fuels must be vaporized before burning, vaporization has been the cornerstone on heat release control. To achieve high vaporization rates, demanded by those devices, the heat transfer from the local atmosphere to the liquid phase must be high. The control of heat transfer may be done by establishing a temperature gradient between the local ambient and the droplet; however, such an approach has limitations. A more versatile way is controlling the contact area of the droplet, which is done by atomization of the liquids with a specified droplet distribution. Under the influence of external magnetic field, the embedded magnetic nanoparticles in the droplets accelerate the droplet heating. This way, the magnetic field modifies the temperature profile in such a way that the boiling condition is found inside the droplet, which can be interpreted as bubble formation and droplet rupture. ${ }^{13,14}$ This way, magnetic hyperthermia may be used as an extra atomization process to control the size of droplets and, accordingly, the vaporization rate of sprays.

This work is organized as follows: in Sec. II, we derive and discuss the model used to determine the heating rate expression. In Sec. III, we apply the model to determine the heating rate for particular types of nanoparticles and external conditions. Finally, in Sec. IV, we present the conclusions.

\section{MODEL AND FORMULATION}

The theoretical model for the heating rate of the ferrofluid droplets is determined by a microscopic model for the magnetic nanoparticles coupled to a thermal reservoir and an external magnetic field. The magnetic nanoparticles are assumed to be monodispersed and monodomain with a spherical shape. Our model may be adapted to polydispersed ferrofluids by replacing the nanoparticle volume by an average volume taken over the distribution of nanoparticles. In this way, this replacement does not change our results in a qualitative way; for this reason, we have chosen to keep the model as simple as possible by considering a monodisperse distribution. A schematic diagram for the system is illustrated in Fig. 1. The uniaxial anisotropy leads to a discrete spectrum for the nanoparticle with an energy barrier separating the two minima. The coupling to the thermal reservoir is mediated by a spin-phonon coupling arising from the nanoparticle crystal field. As discussed in Refs. 28 and 38, the spin-phonon coupling allows the nanoparticle spin to oscillate between the two minima by scaling over the energy barrier. The external magnetic field has two roles in this model: the constant component along the anisotropy axis (taken as the $\hat{z}$-axis) causes an energy imbalance between the energy minima which, combined to the spin-phonon relaxation, leads to an equilibrium situation characterized by a net magnetic moment. The second role is performed by the transversal time-dependent field, which takes the particles back to the higher minimum by providing energy to the nanoparticle. This energy is then transferred to the lattice by spin-phonon coupling. This behavior is analogous to the well-known nuclear magnetic resonance in which the nuclear magnetic moments transfer energy to the lattice absorbed from an external timedependent field. ${ }^{39-42}$ Here, we consider the same process to take place with the difference residing in the energy scale set by the energy barrier from the nanoparticles. In the following, we describe in details each element of the model used to determine the heating rate.

In our study, the contribution of dipolar interactions has been neglected. The ratio of the energy due to dipolar interaction to the energy due to the coupling to the constant field, $\mathrm{B}_{0}$, ranges from $10^{-5}$ to $10^{-6}$ for all the nanoparticles. In this way, this contribution may be safely neglected for these values of concentration of magnetic nanoparticles.

\section{A. Magnetic nanoparticles}

We consider a ferrofluid composed by single-domain magnetic nanoparticles whose fundamental state is described by a $2 \mathrm{~S}+1$ multiplet. It is supposed that the degeneracy is broken by a crystal field and an external magnetic field $\mathbf{B}_{0}=\mathrm{B}_{0} \hat{\mathbf{z}}$, with $\mathrm{B}_{0}$ being a constant. The crystal field has an axial symmetry, being described by a single constant $D$, as follows:

$$
\hat{H}_{S}=-D \hbar^{2} \hat{\sigma}_{z}^{2}-\gamma B_{0} \hbar \hat{\sigma}_{z}
$$

where $\hat{\sigma}_{z}|m\rangle=m|m\rangle$ with $m=-\mathrm{S},-\mathrm{S}+1, \ldots, 0, \ldots \mathrm{S}-1,+\mathrm{S}$; $\gamma$ is the gyromagnetic constant, and $\hbar=h / 2 \pi$, where $h$ is the Planck's constant. This model has been extensively studied in the literature and describes well single-domain magnetic nanoparticles. ${ }^{28,43}$ 

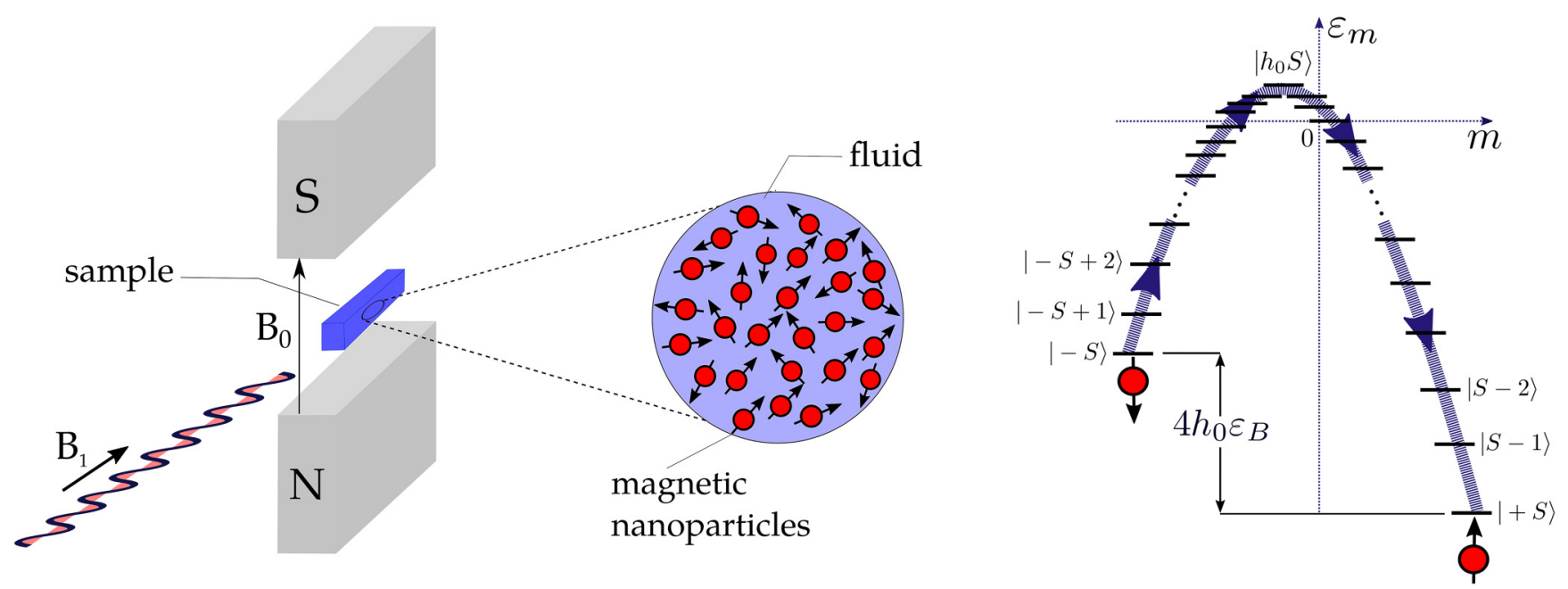

FIG. 1. Schematic diagram of a possible experimental implementation of the system studied in this work. In the left side, the sample with the magnetic fluid is under a vertical magnetic field of constant amplitude, $B_{0}$, provided by the magnets. A transversal time-dependent magnetic field, $\vec{B}_{1}$, is also applied to the sample. These magnetic fields interact with the magnetic moment of the nanoparticles dispersed in the fluid as shown by an enlarged view illustrated in the center of figure. In the right side is shown the $2 S+1$ energy levels for a single nanoparticle. The effect of $B_{0}$ is to introduce an energy imbalance between the two minima, located at the states $|+S\rangle$ and $|-S\rangle$. The temperature and the transverse magnetic field promote the transition among these levels. The inversion of the magnetic moment occurs by climbing up the energy levels as indicated by the blue arrows.

In order to make the notation more compact, we follow the notation employed in Ref. 28 and define the dimensionless constant field, $h_{0}=\gamma \mathrm{B}_{0} / 2 \mathrm{DS} \hbar$. The barrier height is then written as $\varepsilon_{B}=D^{2} \hbar^{2}=\varepsilon_{0} S^{2}$. This gives $\hat{H}_{S}$ the following form:

$$
\hat{\mathrm{H}}_{\mathrm{S}}=-\varepsilon_{0}\left(\hat{\sigma}_{z}^{2}+2 h_{0} \mathrm{~S} \hat{\sigma}_{z}\right) \text {. }
$$

The corresponding eigenvalues of $\hat{H}_{S}$ are then $\varepsilon_{m}=-\varepsilon_{0}\left(m^{2}+2 h_{0} \mathrm{Sm}\right)$. The spectrum defined by $\varepsilon_{m}$ exhibits two minima for $m= \pm S$ and a maximum value for $m=h_{0} S$. As a result, for $h_{0} \neq 0$, there are two energy minima determined by $\varepsilon_{\mathrm{B}}^{ \pm}=\varepsilon_{\mathrm{B}}\left(1 \pm h_{0}\right)$. A plot of Eq. (2) is shown in the right side of Fig. 1.

\section{B. Master equations and relaxation times}

It is considered that the superparamagnetic particles present equal size and a magnetization arising from a large spin $\mathrm{S}$ whose direction relative to the anisotropy axis fluctuates due to an external perturbation which may be due to the presence of phonons as well as a transverse magnetic field. In order to reach the opposite easy direction $-\hat{z}$, the spin must pass through intermediate levels. It worth noting that tunneling processes, which are relevant at low temperatures, are ruled out of the present study. ${ }^{43-48}$

The bare nanoparticle states are described by the Hamiltonian given by Eq. (2). To each state $|m\rangle$, one may write a corresponding master equation for the probability, $p_{m}$, of finding the system in this state. By starting with $m=S$, we have

$$
\dot{p}_{\mathrm{S}}=\mathrm{W}_{\mathrm{S}-1}^{\mathrm{S}} p_{\mathrm{S}-1}-\mathrm{W}_{\mathrm{S}}^{\mathrm{S}-1} p_{\mathrm{S}}
$$

with $\mathrm{W}_{\mathrm{S}-1}^{\mathrm{S}}$ and $\mathrm{W}_{\mathrm{S}}^{\mathrm{S}-1}$ being the transition rates between the energy levels $m=\mathrm{S}$ and $m=\mathrm{S}-1$. The dot over $p$ stands for the time derivative. These transition rates are determined by the Fermi golden rule ${ }^{49}$ from standard perturbation theory.

For intermediate states, the master equation is given by

$$
\dot{p}_{m}=\mathrm{W}_{m-1}^{m} p_{m-1}-\mathrm{W}_{m}^{m-1} p_{m}+\mathrm{W}_{m+1}^{m} p_{m+1}-\mathrm{W}_{m}^{m+1} p_{m},
$$

which takes into account transitions among three levels labeled as $m-k, m$, and $m+k$. The last state, $m=-\mathrm{S}$, is described by

$$
\dot{p}_{-\mathrm{S}}=\mathrm{W}_{-\mathrm{S}+1}^{-\mathrm{S}} p_{-\mathrm{S}+1}-\mathrm{W}_{-\mathrm{S}}^{-\mathrm{S}+1} p_{-\mathrm{S}} .
$$

These equations must be complemented by the condition

$$
p_{-\mathrm{S}}+p_{-\mathrm{S}+1}+\cdots+p_{\mathrm{S}-1}+p_{\mathrm{S}}=1,
$$

which guarantees the probability conservation.

By assuming that the occupation of the intermediate levels is constant, i.e., $d p_{m} / d t=0$ for $m \neq S$, $-\mathrm{S}$, it is possible to write a differential equation for $p_{S}-p_{-S}$

$$
\frac{d\left(p_{S}-p_{-S}\right)}{d t}=-\frac{1}{\tau_{r}}\left(p_{S}-p_{-S}\right) .
$$

Equation (7) is completely general since the nature of the perturbation is determined by the expression for the relaxation rates. All the information about the transition between the multiple levels of the system are encoded within the relaxation time, $\tau_{r}$. To our knowledge, this approach has been first applied by Villain et al. to determine the relaxation time 
of large molecules of Mn-12. ${ }^{38}$ In the following, we consider the coupling to a phonon-bath and an external rotating magnetic field applied to the nanoparticle giving rise to a second relaxation time, $\tau_{f}$. These two parameters allow characterizing the heating rate of ferrofluid droplets. We consider the effect of both perturbations on the system leading to the final expression for the relaxation rate.

A discussion about the precessional movement of the magnetic vector is in order. In fact, Eq. (7) does not contain the precessional motion expected in magnetic systems. This is justified by noting that the precessional motion frequency is much smaller in comparison to the relaxation dynamics for the set of parameters used. In fact, the ratio of the Larmor frequency to the relaxation frequency is determined by $2 h_{0} \eta_{r} / \mathrm{S}$, with $\eta_{r}=\eta_{+} \eta_{-} /\left(\eta_{+}+\eta_{-}\right)$. The expressions for the dimensionless times, $\eta_{+}$and $\eta_{-}$, are presented in the Appendix. This ratio ranges from $10^{-2}$ to $10^{-8}$ for $\mathrm{Fe}_{3} \mathrm{O}_{4}$ to $\mathrm{Fe}$ and $\mathrm{Ni}$, respectively. Hence, the precessional movement may be neglected in this work since the relaxation time is much faster in comparison to the precessional movement.

\section{Spin-phonon relaxation rate}

In this section, we follow the derivation of the spinphonon relaxation rate derived in Ref. 28 by Pfannes et al. In their model, the superparamagnetic particles are considered to present an uniaxial anisotropy whose effect is to produce an energy barrier between two local energy minima. These local energy minima correspond to opposite orientations of the nanoparticle magnetic moment with respect to the easy axis of magnetization. In the presence of the magnetic field, the energy barrier of the nanoparticle will change depending on the orientation of the field, increasing in the sense of the nanoparticle magnetic moment or decreasing in the opposite sense. The coupling of the nanoparticles with a phonon bath introduces a fluctuation in the nanoparticle moment direction with respect to the field and whereby a finite relaxation time, $\tau_{r}$.

The coupling of the spin to the phonon bath is modeled by a spin-phonon Hamiltonian whose origin is borrowed from the early theories of paramagnetic resonance. It is considered that the nanoparticles exhibit a well defined crystalline structure and the magnetic moment from atoms sums up to give rise to the effective total spin, $S$, of the nanoparticle. Each atom is under the influence of the electric field, called crystal field, from the neighboring atoms whose location fluctuates about their equilibrium point. To this fluctuating electric field, there is a magnetic field that interacts with the magnetic moment at the rest frame of the atom causing its relaxation. The crystal field is expressed in terms of spherical harmonics and then mapped into angular momentum operators. The effect of temperature is taken into account by modulating the crystal field by a strain operator, accounting for the crystal deformation, which is then expressed in terms of phonon field.

Within the description of the previous paragraph, it is possible to build an effective spin-phonon Hamiltonian in order to describe the coupling of spin degrees of freedom to the thermal reservoir. In Ref. 28, the spin-phonon interaction was modeled by using the following expression:

$$
\hat{\mathrm{H}}_{s p-p h}=\sum_{j} \hat{\mathrm{S}}(j) \varepsilon_{a v}\left(\mathbf{r}_{j}\right)
$$

where $\hat{S}(j)$ is the spin operator acting on states defined in Eq. (2). In general, the operator $\hat{S}(j)$ is written as a series of crystal field operators carrying the information about the geometry of the lattice. The factor $\varepsilon_{a v}\left(\mathbf{r}_{j}\right)$ is the average strain operator evaluated at the position $\mathbf{r}_{j}$ of the nanoparticle crystal lattice. Explicitly, the average strain operator reads

$$
\varepsilon_{a v}=\sum_{j} \mathrm{Q}_{j} k_{j}\left[\hat{a}_{j}^{\dagger} \exp \left(-i \mathbf{k} \cdot \mathbf{r}_{j}\right)+\hat{a}_{j} \exp \left(i \mathbf{k} \cdot \mathbf{r}_{j}\right)\right],
$$

in which $Q_{j}=\left(\hbar / 2 M \omega_{j}\right)^{1 / 2}$, with $M$ being the crystal mass, $k_{j}$ and $\omega_{j}$ are the wavevector and angular frequency, and $\hat{a}_{j}^{\dagger}$ and $\hat{a}_{j}$ are the creation and annihilation operators for the particular phonon mode $j$. The sum over $j$ in Eq. (8) leads to a mixture of magnetic and thermal degrees of freedom.

The spin-phonon Hamiltonian acts on a combined spinphonon state $|m\rangle \otimes\left|n_{1}, \ldots, n_{j}, \ldots\right\rangle$ with $m$ being defined in Eq. (2) and $n_{j}$ is the number of phonons in the mode $j$. The coupling produces the transition rates appearing in the master equations [Eqs. (3)-(6)]. By using standard time perturbation theory, one can determine each transition rate appearing in those equations and determine the relaxation time, $\tau_{r}$. The final expression is discussed in the Appendix and a more detailed account may be found in Ref. 28 .

It is worth mentioning that we have chosen a quantum approach which leads to the description of details of the relaxation process in order to correlate such a process with microscopic parameters from the magnetic nanoparticles. This approach may be related with previous works ${ }^{50-52}$ in which the relaxation is treated classically by the replacement $\hat{\sigma}_{z} \rightarrow \mathrm{S} \cos \theta$. Besides the quantum description of the relaxation, our approach may be used to estimate the quantum tunneling contribution in low temperatures (not considered in this work).

\section{External magnetic field interaction}

It is also considered the presence of a transverse magnetic field, of amplitude $B_{1}$, whose form is given by

$$
\mathbf{B}_{1}(t)=\sum_{j}\left(B_{1} \cos \omega_{j} t \hat{\mathbf{x}}+B_{1} \sin \omega_{j} t \hat{\mathbf{y}}\right),
$$

where $\omega_{j}$ is the angular frequency of the field. It is supposed that the magnetic field exhibits a finite bandwidth of frequencies which is represented by the sum over $j$. The nanoparticle magnetic moment couples to this field leading to a relaxation-like process where the magnetic moment of the nanoparticles tends to be aligned to the magnetic field. We assume the coupling of the nanoparticle to the transverse 
field is determined by

$$
\hat{\mathrm{H}}_{1}=\sum_{j} \frac{\hbar \gamma \mathrm{B}_{1}}{2}\left(e^{i \omega_{j} \mathrm{t}} \hat{\sigma}_{-}+e^{-i \omega_{j} \mathrm{t}} \hat{\sigma}_{+}\right) .
$$

The Hamiltonian given by Eq. (10) is used as the perturbation allowing the transition among the states of Eq. (1). The transitions among these levels are described by the same set of master equations defined by Eqs. (3)-(6). By using standard time-dependent perturbation theory, ${ }^{49}$ each transition rate appearing in those equations is calculated. By assuming the intermediate levels are stationary, it is possible to obtain an analogous expression to Eq. (7) with a new characteristic time, $\tau_{f}$, encoding all transitions among the intermediate levels. These transitions occur by resonance with the transverse field whose frequency coincides to the levels spacing. For this to occur, it is necessary that the transverse field should contain all the frequencies for the different spacing energies between the adjacent levels. In this way, we assume a finite and continuous band of frequencies described by a Lorentzian function as follows:

$$
f(\hbar \omega)=\frac{\delta / \pi}{\hbar^{2} \omega^{2}+\delta^{2}},
$$

with $\delta$ being the halfwidth of the band. Equation (11) allows converting the discrete sum appearing in Eqs. (9) and (10) into an integral over the frequency.

By using the general master equations given by Eqs. (3)-(6), one is able to determine the relaxation time $\tau_{f}$ due to the transversal field. Since the calculations are rather standard, we present the final expression for $\tau_{f}$

$$
\tau_{f}=\tau_{\mathrm{B}} \eta_{f}
$$

with

$$
\eta_{f}=\frac{1}{4 \delta^{\star} h_{1}^{2}}\left[\zeta_{f}\left(+h_{0}\right)+\zeta_{f}\left(-h_{0}\right)\right]
$$

and

$$
\zeta_{f}\left( \pm h_{0}\right)=\sum_{j=0}^{S\left(1 \pm h_{0}\right)-1} \frac{(2 j+1)^{2}+\delta^{\star 2}}{\left[S\left(1 \mp h_{0}\right)+j+1\right]\left[S\left(1 \pm h_{0}\right)-j\right]}
$$

Notice that the characteristic time of the barrier $\tau_{\mathrm{B}}=\hbar / \varepsilon_{\mathrm{B}}$ and a dimensionless linewidth $\delta^{*}=\delta \mathrm{S}^{2} / \varepsilon_{\mathrm{B}}$ were defined. In order to measure the intensity of the transverse field, we also have defined the dimensionless parameter $h_{1}=\gamma \hbar B_{1} S / 2 \varepsilon_{B}$.

Owing to a microscopic model involving many processes, this model involves many energies scales. Besides the parameters $h_{0}$ and $h_{1}$, related to the amplitude of the static and transverse fields, respectively, the heating rate is also dependent on the linewidth $\delta^{*}$ and the energy spacing among the levels of the magnetic nanoparticle. All these parameters are mixed, within the master equations, to give a global effect of heating. In this way, one cannot isolate the contribution of the magnetic energy, coming from the fields, in a single parameter in order to compare to the thermal energy delivered as heat to the fluid. As a result, it is not possible to determine conversion efficiency of the heating process as a whole.

\section{Heating rate equations}

In Secs. II A and II B, the relaxation times, $\tau_{r}$ and $\tau_{f}$, have been determined by assuming the contributions from the thermal reservoir and the transverse field separately. Now, we consider the combined effect of both contributions in order to develop an expression for the heating rate of magnetic nanoparticles.

By assuming that the intermediate levels are stationary, we focus on the $\pm S$ states and treat the nanoparticle as a two level system with an energy gap equal to $4 h_{0} \varepsilon_{\mathrm{B}}$. By denoting the number of magnetic nanoparticles in the states $+\mathrm{S}$ and $-\mathrm{S}$ as $\mathrm{N}_{+}$and $\mathrm{N}_{-}$, respectively, the time variation of these two numbers is determined by the following set of equations:

$$
\frac{d \mathrm{~N}_{ \pm}}{d t}=\frac{\mathrm{N}_{\mp}}{\tau_{\mp}}-\frac{\mathrm{N}_{ \pm}}{\tau_{ \pm}}+\frac{1}{\tau_{f}}\left(\mathrm{~N}_{\mp}-\mathrm{N}_{ \pm}\right)
$$

Equation (15) may be combined by defining $n=\mathrm{N}_{-}-\mathrm{N}_{+}$ and $\mathrm{N}=\mathrm{N}_{-}+\mathrm{N}_{+}$,

$$
\frac{d n}{d t}=\frac{n_{0}-n}{\tau_{r}}-\frac{2 n}{\tau_{f}}
$$

with $n_{0}=\mathrm{N}\left[\left(\eta_{-}-\eta_{+}\right) /\left(\eta_{+}+\eta_{-}\right)\right]$and $\tau_{r}=\tau_{\mathrm{B}} \eta_{r}$ with $\eta_{r}=\eta_{+} \eta_{-} /$ $\left(\eta_{+}+\eta_{-}\right)$. By assuming the stationary case $d n / d t=0$, we obtain

$$
n=\frac{n_{0}}{1+2 \eta_{r} / \eta_{f}} .
$$

The rate at which the energy due to the external field is provided to the nanoparticles may be computed by means of a detailed balance equation

$$
\frac{d \varepsilon}{d t}=\frac{4 h_{0} \varepsilon_{B}}{\tau_{f}}\left(N_{-}-N_{+}\right)
$$

where $4 h_{0} \varepsilon_{B}$ is the energy difference between $+S$ and $-S$ states. Since $N_{-}-N_{+}=n$, one may use Eq. (17) together with the definition of $n_{0}$ to write Eq. (18) as

$$
\frac{d \varepsilon}{d t}=\frac{4 \mathrm{~N} \varepsilon_{\mathrm{B}}}{\tau_{\mathrm{B}}} h_{0}\left[\frac{\eta_{+}-\eta_{-}}{\eta_{+} \eta_{f}+2 \eta_{-} \eta_{+}+\eta_{-} \eta_{f}}\right]
$$

In order to express the heating rate due to the field, we use the concentration of nanoparticles along with the thermal properties of the ferrofluid as the specific heat and mass density. ${ }^{53}$ In this way, we write

$$
\frac{\Delta \mathrm{T}}{\Delta \mathrm{t}}=\frac{1}{\mathrm{~V} \xi} \frac{d \varepsilon}{d t}
$$

with $\mathrm{V}$ being the volume, $\xi=(1-\varphi) \mathrm{c}_{\mathrm{F}} \rho_{\mathrm{F}}+\varphi \mathrm{C}_{\mathrm{P}} \rho_{\mathrm{P}}$ with $\mathrm{c}_{\mathrm{F}}\left(\mathrm{c}_{\mathrm{P}}\right)$ being the specific heat capacity of the fluid (nanoparticle), while $\rho_{\mathrm{F}}\left(\rho_{\mathrm{P}}\right)$ is the mass density of the fluid (nanoparticle). The parameter $\varphi$ is the volume fraction determining the concentration of the nanoparticles embedded in the fluid. 
Substitution of Eq. (19) into Eq. (20) leads to the heating rate expression

$$
\frac{\Delta \mathrm{T}}{\Delta \mathrm{t}}=4 \mathrm{Ch} 0\left[\frac{\eta_{+}-\eta_{-}}{\eta_{+} \eta_{f}+2 \eta_{-} \eta_{+}+\eta_{-} \eta_{f}}\right],
$$

with the constant $\mathrm{C}$ being defined as

$$
\mathrm{C}=\frac{\mathrm{N} \varepsilon_{\mathrm{B}}}{\mathrm{V} \xi_{\mathrm{B}}}
$$

which sets the heating rate scale. In fact, the constant $C$ is determined by both the fluid and the nanoparticle properties. The ratio $\mathrm{N} / \mathrm{V}$ is the density of particles within the ferrofluid and may be expressed in terms of the volume fraction, $\varphi$. In fact, one can write

$$
\frac{\mathrm{N}}{\mathrm{V}}=\frac{\varphi}{\mathrm{V}_{\mathrm{P}}}
$$

where $V_{P}$ is the volume of the nanoparticle.

We notice that the main parameter in order to characterize the heating rate is the energy barrier, $\varepsilon_{\mathrm{B}}$. In this way, it is necessary to access such a parameter in experiments. This is done by first noting that $\varepsilon_{B}$ may be related to the anisotropy energy and the volume of the nanoparticle. One can write $\varepsilon_{\mathrm{B}}=D \hbar^{2} \mathrm{~S}^{2}=\mathrm{K} \mathrm{V}_{\mathrm{P}}$, with $\mathrm{K}$ being the anisotropy constant and $\mathrm{V}_{\mathrm{P}}$ the nanoparticle volume. The Stoner-Wohlfarth model ${ }^{70}$ allows the product $K V_{P}$ to be related to the blocking temperature, $\theta_{\mathrm{B}}$, whose value may be determined, for instance, in magnetization measurements. Once the nanoparticles are assumed to have a single domain structure with uniaxial anisotropy, one can show that the energy barrier may be written as ${ }^{57}$

$$
\varepsilon_{\mathrm{B}}=25 k_{\mathrm{B}} \theta_{\mathrm{B}} \text {. }
$$

Since there are many experimental data involving blocking temperature and the anisotropy energy, we perform a connection between the microscopic formalism developed above and classical approach due to Stoner-Wohlfarth. However, it is worth noting that as long as the energy barrier, spin-phonon coupling, and the other microscopic parameters are available in the literature, it is possible to compare the model directly to experiments without using the Stoner-Wohlfarth model.

Equations (21), (23), and (24) allow one to determine the heating rate from a knowledge of the ferrofluid properties. In the following, we analyze Eq. (21) as a function of the main parameters characterizing the model.

\section{RESULTS AND DISCUSSION}

In this section, we apply the model defined by Eqs. (21), (23), and (24) to study the heating rate in a magnetic fluid seeking for its application in fuel combustion. In order to predict realistic situations, we use the values obtained in the literature for different magnetic nanoparticles, as listed in Table I. We have considered five different magnetic nanoparticles characterized by their composition, blocking temperature, $\theta_{\mathrm{B}}$, and diameter, $d$. The corresponding specific heat, $c_{\mathrm{P}}$, and mass density, $\rho_{\mathrm{P}}$, for each material is also shown in Table I. We have focused on nanoparticles with a diameter smaller than $10 \mathrm{~nm}$ in which the Néel process is the predominant mechanism of relaxation. The energy barrier, $\varepsilon_{B}$, was determined by using Eq. (24). We have expressed such values in units of temperature by using $\varepsilon_{\mathrm{B}} / k_{\mathrm{B}}$. It is assumed that the nanoparticles must be immersed in fuel droplets whose typical diameter is $\sim 10^{-5} \mathrm{~m}$. This corresponds to the phase of vaporization of the fuel to be used in the combustion process. In this way, one expects a highly diluted limit in order to maintain the fuel parameters as close to their nominal values as possible. Under this condition, we have used a volume fraction of $\varphi=0.005 \%$ which leads to about $10^{5}$ to $10^{6}$ nanoparticles within a single droplet for diameters ranging from 7 to $2 \mathrm{~nm}$.

In order to estimate the nanoparticle spin, $\mathrm{S}$, the values $\mathrm{V}_{\mathrm{cl}}$ and $\mu_{\mathrm{cl}}$ for the volume and magnetic moment of each unit cell have been used. These values were determined from the literature and are listed in Table I. The total spin is then given by $\mathrm{S}=\mu_{\mathrm{cl}} \mathrm{V}_{\mathrm{P}} / g_{a v} \mathrm{~V}_{c l}$, where $\mu_{\mathrm{cl}}$ is expressed in Bohr magneton units and $g_{a v}$ is the average g-factor, whose value was taken equal to 2 as an approximation. The spin $S$ is used to predict the frequency band of the transversal field. The minimum and maximum values for the frequency have been determined by using the smallest and largest values of the energy spacing between the levels of $\hat{H}_{S}$. These values are given by $f_{\min }=25 k_{\mathrm{B}} \theta_{\mathrm{B}} / h \mathrm{~S}^{2}$ and $f_{\max }=25 k_{\mathrm{B}} \theta_{\mathrm{B}}\left[2 \mathrm{~S}\left(1-h_{0}\right)-1\right] / h \mathrm{~S}^{2}$, with $h$ being the Planck constant.

The spin-phonon coupling constant, $h_{s p}$, is the parameter describing the coupling of the magnetic moment to the phonon bath. The value of $h_{s p}=10^{-2}$, used in Figs. 3 and 4 , gives an estimate of the order of magnitude of this constant. In fact, this value corresponds to the one obtained for Mn-ferrite nanoparticles, ${ }^{71}$ by Mössbauer measurements, and we have considered that such a value must be of the same order of magnitude for all nanoparticles studied in this work. In order to justify such an estimate, we have studied the variation of the heating rate with the transverse field, $B_{1}$, for different values of the spin-phonon coupling. We have taken $h_{s p}$ as a free parameter and analyzed its variation around the experimental value of $10^{-2}$. The corresponding heating rate curves are shown in Fig. 2. For $h_{s p}>10^{2}$, one notices that the heating rate is suppressed. Actually, for $h_{s p}=10^{2}$ (yellow solid curve), the heating rate is smaller than $10^{-4} \mathrm{~K} / \mathrm{s}$. On the other hand, when $h_{s p}$ is reduced to values smaller than $10^{-2}$, the heating rate increases slightly but saturates as $h_{s p}$ is reduced beyond $10^{-3}$. In fact, the three curves for $h_{s p}$ ranging from $10^{-3}$ to $10^{-6}$ are superimposed to each other. In this way, the value of $10^{-2}$ used in this work is a reasonable estimate of $h_{s p}$.

The fluid properties enter into the heating rate formula through the specific heat and mass density appearing in the constant C, Eq. (22). These data for most common fuels are also listed in Table I. With all these data, one is able to study the heating rate with the variation of external parameters like the temperature and the amplitude of the applied magnetic field. In this respect, we have considered both, the constant and the alternate transverse component, to have an amplitude up to a maximum of $0.5 \mathrm{~T}$. In fact, these values may be easily obtained in experiments. In addition, the temperature 
TABLE I. Typical magnetic parameters used in the heating rate estimate used in this work.

\begin{tabular}{|c|c|c|c|c|c|c|c|c|}
\hline \multicolumn{9}{|c|}{ Nanoparticle parameters } \\
\hline Material & $\begin{array}{l}\theta_{B}{ }^{a} \\
(K)\end{array}$ & $\begin{array}{c}d^{a} \\
(\mathrm{~nm})\end{array}$ & $\begin{array}{l}\varepsilon_{B}{ }^{b} \\
(K)\end{array}$ & $\begin{array}{c}c_{P}{ }^{c} \\
(\mathrm{~J} / \mathrm{kg} \mathrm{K})\end{array}$ & $\begin{array}{c}\rho_{P}{ }^{\mathrm{d}} \\
\left(\mathrm{kg} / \mathrm{m}^{3}\right)\end{array}$ & $\begin{array}{l}\mu_{c l}{ }^{e} \\
\left(\mu_{B}\right)\end{array}$ & $\begin{array}{l}V_{c b_{3}^{f}}^{f} \\
\left(A^{\prime}\right)\end{array}$ & $\begin{array}{c}\boldsymbol{f}_{\min }-\boldsymbol{f}_{\max }{ }^{g} \\
(\mathbf{G H z})\end{array}$ \\
\hline $\mathrm{Fe}$ & 12 & 2.0 & 300 & 447 & 7860 & 2.22 & 23.64 & $0.16-35$ \\
\hline Co & 73 & 3.3 & 1825 & 423 & 8900 & 1.72 & 25.64 & $0.09-93$ \\
\hline $\mathrm{Ni}$ & 14 & 3.8 & 350 & 444 & 8900 & 0.605 & 43.61 & $0.18-45$ \\
\hline $\mathrm{Fe}_{3} \mathrm{O}_{4}$ & 203 & 6.6 & 5075 & 670 & 5180 & 4.10 & 575.9 & $0.37-367$ \\
\hline$\gamma-\mathrm{Fe}_{2} \mathrm{O}_{3}$ & 94.3 & 3.0 & 2357 & 746 & 4600 & 2.50 & 580.1 & $0.54-3193$ \\
\hline fcc FePt & 20 & 4.0 & 500 & 327 & 15200 & 0.63 & 56.62 & $0.30-84$ \\
\hline \multicolumn{9}{|c|}{ Basic-fluid parameters } \\
\hline Fluid & $\begin{array}{c}c_{F}^{h} \\
(\mathrm{~J} / \mathrm{kg} \mathrm{K})\end{array}$ & $\underset{\left(\mathrm{kg} / \mathrm{m}^{3}\right)}{\rho_{F}^{h}}$ & & & & & & \\
\hline Kerosene & 2080 & 840 & & & & & & \\
\hline Gasoline & 2400 & $720-780$ & & & & & & \\
\hline Light diesel & 2200 & $780-840$ & & & & & & \\
\hline Benzene & 1720 & 879 & & & & & & \\
\hline Methanol & 2600 & 792 & & & & & & \\
\hline Ethanol & 2500 & 785 & & & & & & \\
\hline
\end{tabular}

${ }^{\text {aT The }} \theta_{B}$ and $d$ values were obtained from Ref. 54 for Fe; Ref. 55 for Co; Ref. 56 for Ni; Ref. 57 for $\mathrm{Fe}_{3} \mathrm{O}_{4}$; Ref. 58 for $\gamma-\mathrm{Fe}_{2} \mathrm{O}_{3}$; Ref. 59 for fcc-FePt.

${ }^{\mathrm{b}}$ The $\varepsilon_{B}$ values were calculated by using Eq. (24) and expressed in units of temperature, i.e., $\varepsilon_{B} / k_{B}=25 \theta_{B}$.

${ }^{C}$ The $c_{P}$ bulk values were obtained from Ref. 60 for Ni; Ref. 61 for $\mathrm{Fe}_{3} \mathrm{O}_{4}, \gamma-\mathrm{Fe}_{2} \mathrm{O}_{3}, \mathrm{FeCo}$, and fcc FePt; Ref. 62 for Fe and Co.

${ }^{\mathrm{d}}$ The $\rho_{P}$ bulk values were obtained from Ref. 61 for $\mathrm{Fe}_{3} \mathrm{O}_{4}, \gamma-\mathrm{Fe}_{2} \mathrm{O}_{3}$, FeCo, and fcc FePt; Ref. 62 for Ni, Fe, and Co.

${ }^{\mathrm{e}}$ The magnetic moment per unit cell, $\mu_{c l}$, is expressed in Bohr magneton units, $\mu_{B}=9.27 \times 10^{-24} \mathrm{~J} / \mathrm{T}$. The values for Fe, $\mathrm{Co}, \mathrm{Ni}$, and $\mathrm{Fe}_{3} \mathrm{O}_{4}$ were obtained from Ref. 63 . The values for $\gamma-\mathrm{Fe}_{2} \mathrm{O}_{3}$ and fcc FePt were obtained from Refs. 64 and 65, respectively.

fUnit cell volume, $V_{c l}$. The values for $\mathrm{Fe}$, $\mathrm{Co}$, and $\mathrm{Ni}$ were obtained from Ref. 66; the data for $\mathrm{Fe}_{3} \mathrm{O}_{4}, \gamma-\mathrm{Fe}_{2} \mathrm{O}_{3}$, and fcc-FePt were obtained in Refs. 67,64, and 65.

${ }^{g}$ Calculated values of the frequency range for a fixed magnetic field of $B_{0}=1 \mathrm{~T}$. The minimum value of the frequency was determined by $f_{\min }=\frac{25 k_{B} \theta_{B}}{h S^{2}}$, while the maximum value was determined by $f_{\max }=\frac{25 k_{B} \theta_{B}}{h S^{2}}\left[2 S\left(1-h_{0}\right)-1\right]$.

${ }^{\mathrm{h}}$ The values of $c_{F}$ and $\rho_{F}$ for kerosene were obtained from Ref. 68. The data for the other fuels were extracted from Ref. 69. These values are valid for all fuels in the liquid phase under $0^{\circ} \mathrm{C}$ and $1 \mathrm{~atm}$.

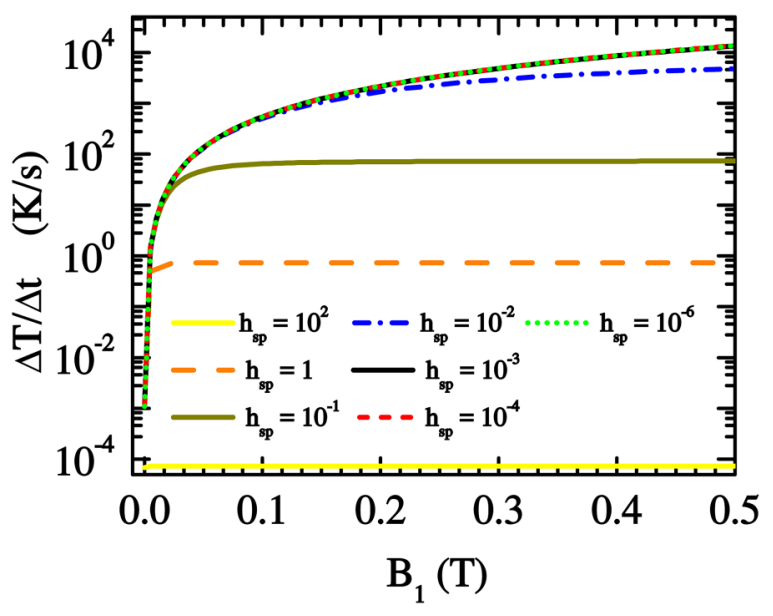

FIG. 2. Heating rate curves for different values of spin-phonon energy, $h_{s p}$. The spin-phonon values range from $10^{-6}$ to $10^{2}$, and one notices that the highest values of $\Delta T / \Delta t$ are obtained for $h_{s p}$ ranging from $10^{-2}$ to $10^{-6}$. All curves were calculated for fixed parameters: $T=300 \mathrm{~K}, B_{0}=0.5 \mathrm{~T}, \varphi=0.005 \%$. was fixed within a range around room temperature fixed at $300 \mathrm{~K}$.

In Fig. 3(a), the heating rate as a function of the transverse field amplitude, $B_{1}$, is shown for different values of temperature. All the curves were determined by assuming nanoparticles of $\mathrm{Fe}_{3} \mathrm{O}_{4}$ under a fixed magnetic field of $\mathrm{B}_{0}=0.1 \mathrm{~T}$. For all temperatures, the same trend is observed, with an abrupt increase with $B_{1}$ and then a saturation for larger values of $B_{1}$. For $T=175 \mathrm{~K}$, the saturation occurs for fields above $0.01 \mathrm{~T}$, while for $\mathrm{T}=300 \mathrm{~K}$, the saturation shifts to fields above $\sim 0.14 \mathrm{~T}$. For temperatures above $300 \mathrm{~K}$, the saturation occurs for fields above $0.5 \mathrm{~T}$. The maximum value of the heating rate increases with temperature as evident by comparing the curves of Fig. 3(a). In fact, as temperature increases, the number of phonons increases accordingly helping the relaxation process to take place. As a result, the absorption of heat from the transverse field is more efficient.

The effect of the fixed magnetic field, $B_{0}$, on the heating rate is considered in Fig. 3(b). All curves were determined at the same temperature $\mathrm{T}=300 \mathrm{~K}$. One observes the same trend as found in Fig. 3(a) with an abrupt increase and then a plateau where the heating rate attains its maximum value. 

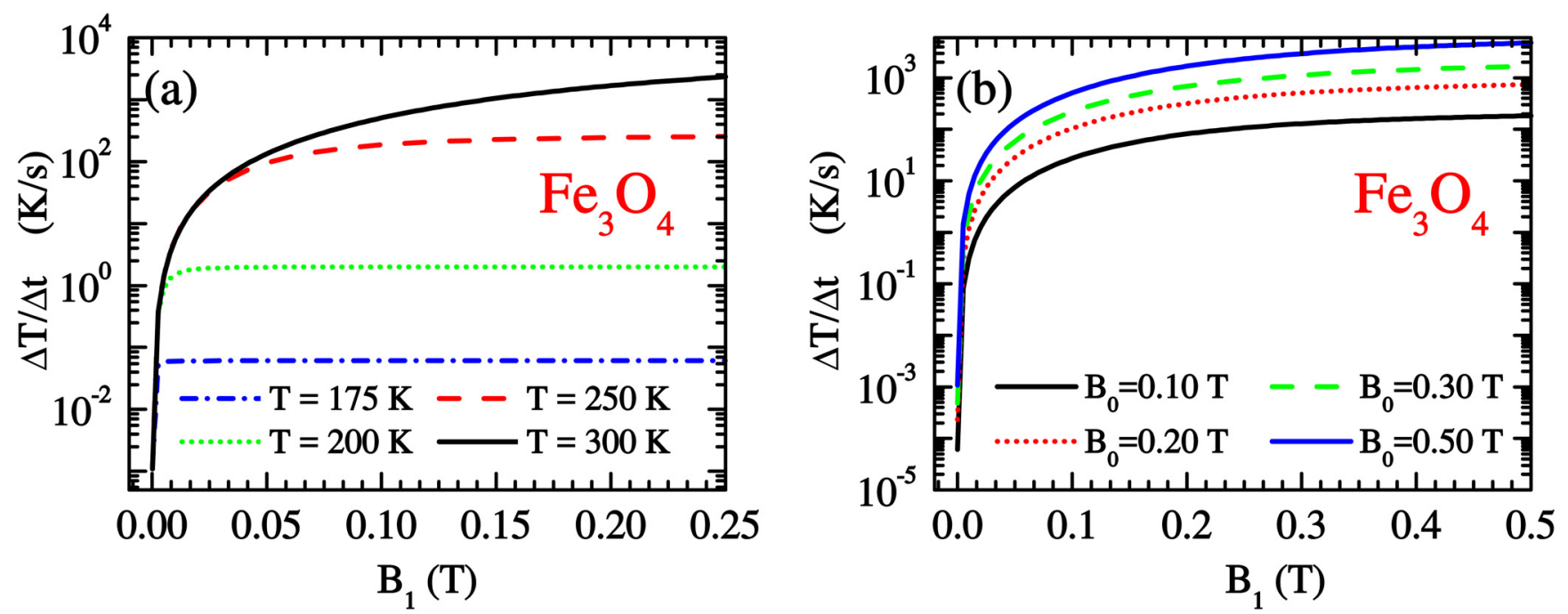

FIG. 3. The heating rate [Eq. (21)] as a function of the transverse field amplitude, $B_{1}$. (a) Curves for different values of temperature with the constant field amplitude, $B_{0}=0.1 \mathrm{~T}$. The parameters used are the ones of Table I for $\mathrm{Fe}_{3} \mathrm{O}_{4}$ and kerosene. (b) Heating rate for different values of the constant field, $B_{0}$, with temperature $T=300 \mathrm{~K}$. All graphs in this panel were calculated by using spin-phonon energy and the volume fraction equal to, respectively, $h_{s p}=10^{-2}$ and $\varphi=0.005 \%$.

The increase of $B_{0}$ pushes the plateau to higher values. In fact, it is changed from $220 \mathrm{~K} / \mathrm{s}$ for $\mathrm{B}_{0}=0.1 \mathrm{~T}$ to $6300 \mathrm{~K} / \mathrm{s}$ for $B_{0}=0.5 \mathrm{~T}$. This increase is related to the energy difference between the states $+S$ and $-S$, which is proportional to $B_{0}$ as one can observe from Eq. (21). This energy difference is absorbed from the transverse field by the nanoparticles and converted into heat by the coupling to the phonon bath. In contrast to the results of Fig. 3(a), the saturation field seems to be less dependent on $B_{0}$. By comparing the curves of Fig. $3(\mathrm{~b})$, one observes that all curves saturate for $\mathrm{B}_{1}>0.14 \mathrm{~T}$.

The effect of the external variables like temperature and field were addressed in Figs. 3(a) and 3(b). Now, we consider the effect of the materials on the heating rate. In Fig. 4(a), we consider the heating rate for different nanoparticles listed in Table I. We have used $\mathrm{T}=300 \mathrm{~K}$ and $\mathrm{B}_{0}=0.1 \mathrm{~T}$ as fixed parameters. All curves follow the same trend as previously
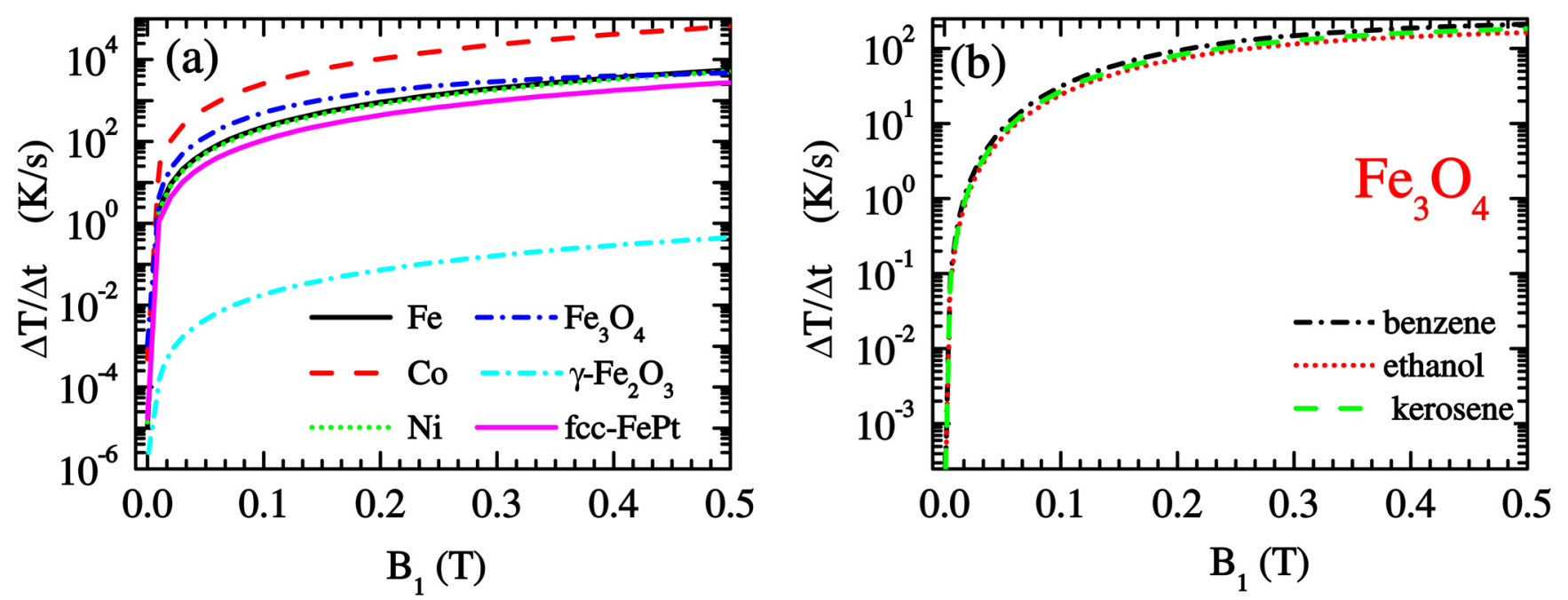

FIG. 4. The heating rate [Eq. (21)] as a function of the transverse field amplitude, $B_{1}$. (a) Heating rate for different nanoparticles listed in Table I with $T=300 \mathrm{~K}$ and $B_{0}=0.1 \mathrm{~T}$. It is considered all the nanoparticles immersed in kerosene. (b) $\mathrm{A}$ comparison of the heating rate for nanoparticles of Fe $\mathrm{O}_{4}$ immersed in different fuels. All curves were determined with $T=300 \mathrm{~K}$ and $B_{0}=0.1 \mathrm{~T}$. Both graphs in this panel were calculated by using spin-phonon energy and the volume fraction equal to, respectively, $h_{s p}=10^{-2}$ and $\varphi=0.005 \%$. 
observed, but the plateau and the saturation field are different for each nanoparticle. The highest heating rate is observed for Co nanoparticles whose maximum reaches a rate around $\sim 10^{4} \mathrm{~K} / \mathrm{s}$. The lowest rate was $10^{-1} \mathrm{~K} / \mathrm{s}$ observed for $\gamma-\mathrm{Fe}_{2} \mathrm{O}_{3}$. This was obtained for the maximum value of the $B_{1}$ field. The discrepancy among all nanoparticles reveals the need to take into consideration their microscopic properties in order to project systems more efficiently. The heating rate is dependent on all properties of the nanoparticles including their physical size and magnetic properties. All these properties combined give origin to the heating curve appearing in Fig. 4(a). In this way, it is not possible to draw any conclusion based on a single parameter like the energy barrier or nanoparticle volume.

In Fig. 4(b), we have tested three fuels in which $\mathrm{Fe}_{3} \mathrm{O}_{4}$ are immersed. There is no significant difference on the heating rate observed for these fuels. In fact, the information about the fuels enters into the model through their mass density and specific heat. As shown in Table I, these values are close to each other which leads to curves of Fig. 4(b).

The results presented are in accordance with previous reports in the literature. In fact, the heating rates obtained in this work have the same order of magnitude as reported in Refs. 25 and 53. It is worth noting that the application of magnetic hyperthermia on fuel combustion, considered in this work, is relatively new, and experimental studies are necessary in order to establish a systematic validation of our model.

\section{CONCLUSION}

The behavior of the heating rate for magnetic fluids envisaging their application in fuel combustion was studied. The combustion phenomenon relies on the vaporization of combustion fuels in small droplets in order to achieve high temperatures required to the burning of the droplets. As a result, the combustion engines exhibit big sizes to comply with the large volumes required by the fuels in the vaporized state. It has been shown that the use of magnetic nanoparticles, embedded into the fuel, may lead to the achievement of heating rates of the order of $10^{3} \mathrm{~K} / \mathrm{s}$. Such values may contribute to a significant volume reduction of combustion chambers. This may lead to the construction of compact combustion engines, considerably lighter in comparison to the current technology, which may save the costs in their construction and operation.
In order to maximize the heating rate, one must choose the appropriate material for the magnetic nanoparticles. Under the same external conditions, we have found significant differences between the maximum heating rate obtained for different nanoparticles. In fact, it varies about four orders of magnitude among the nanoparticles used in this study. Besides, we show that the heating rate can be optimized at high values for certain values of temperature and magnetic field. Also, we find that these results are robust against the hosting fuel.

In this way, a microscopic approach taking into account the relaxation process occurring within the nanoparticles may help to project systems with a better performance in magnetic hyperthermia.

\section{ACKNOWLEDGMENTS}

E.C.S. acknowledges support from the CNPq agency under Grant No. 307466/2016-7. P.A.O. acknowledges financial support from FONDECYT under Grant No. 1140571. F.F.F. and E.C.S. acknowledge CAPES agency financial support (No. CAPES PNPD 028/2010).

\section{APPENDIX: THERMAL RELAXATION TIME}

The expression for the relaxation time, obtained in Ref. 28, is given by

$$
\tau_{r}^{-1}=\tau_{+}^{-1}+\tau_{-}^{-1}
$$

with

$$
\tau_{ \pm}=\tau_{\mathrm{B}} \eta_{ \pm}
$$

The relaxation time, $\tau_{r}$, is determined in terms of $\tau_{+}$, corresponding to the time the nanoparticle takes to climb the barrier from $-\mathrm{S}$ to $+\mathrm{S}$ and $\tau_{-}$, corresponding to the opposite movement. The energy barrier defines a natural time scale defined by $\tau_{\mathrm{B}}=h / \varepsilon_{\mathrm{B}}$. The dimensionless time $\eta_{ \pm}$ is written as follows:

$$
\eta_{ \pm}=h_{s p}^{2} \exp \left(\alpha_{ \pm}\right)\left[\zeta\left(+h_{0}\right)+\zeta\left(-h_{0}\right)\right]
$$

and

$$
\zeta\left( \pm h_{0}\right)=\left(\frac{S}{2}\right)^{6(\mathrm{~S} / 2)\left(1 \pm h_{0}\right)-1} \frac{\exp \left[-\alpha\left(\frac{2}{S}\right)^{2} i^{2}\right]}{(1+2 i)^{3}\left[\mathrm{~S}\left(1 \pm h_{0}\right)-2 i\right]\left[\mathrm{S}\left(1 \pm h_{0}\right)-2 i-1\right]} \frac{1-\exp \left[-\alpha\left(\frac{2}{S}\right)^{2}(1+2 i)\right]}{\left.\left.\mathrm{S}\left(1 \mp h_{0}\right)+2 i+2\right)\right]\left[\mathrm{S}\left(1 \mp h_{0}\right)+2 i+1\right]}
$$

where the constants $\alpha, h_{0}$, and $h_{s p}$ are given by

$$
\begin{gathered}
\eta_{ \pm}=\alpha\left(1 \pm h_{0}\right)^{2}, \quad \alpha=\frac{\varepsilon_{\mathrm{B}}}{k_{\mathrm{B}} \mathrm{T}}, \quad h_{0}=\frac{\gamma \hbar \mathrm{B}_{0} \mathrm{~S}}{2 \varepsilon_{\mathrm{B}}}, \quad \text { and } \\
h_{\mathrm{sp}}=\frac{\varepsilon_{s p}}{\varepsilon_{\mathrm{B}}} .
\end{gathered}
$$

Notice that all the parameters in Eq. (4) are expressed in terms of the energy barrier, $\varepsilon_{B}$, taken as the natural scale of the problem. The parameter $\alpha$ is the ratio of the anisotropy energy $K V_{P}$ to the thermal energy $k_{B} T$, with $K$ being the anisotropy constant and $V_{P}$ the particle volume. In the absence of an external field, $K V_{P}$ gives an estimate of the height of the 
energy barrier between the two energy minima. In the limit of high temperatures $(T \rightarrow \infty)$, the energy barrier is too small in comparison to the thermal energy and, as a result, the magnetic moment is randomized, which is known as superparamagnetic limit. Conversely, when the temperature is close to zero, the magnetic moment is fixed, since there is no enough energy to overcome the barrier, and the particle is said blocked. When a magnetic field is applied, it is introduced an asymmetry between the two energy minima which is described by the parameter $h_{0}$, ranging between 0 and 1 . It is defined by the ratio of the magnetic energy $\left(g \mu_{\mathrm{B}} \mathrm{H}_{0} \mathrm{~S}\right)$ to the anisotropy energy $\mathrm{KV}_{\mathrm{P}}$, where $g$ is the well known $g$-factor and $\mu_{\mathrm{B}}$ is the Bohr magneton. The asymmetry between these two minima increases with the applied field until some limit value where one of these minima coincides with the top of the barrier. In this case, the relaxation is quenched and the system becomes saturated. This limit field occurs when $g \mu_{\mathrm{B}} \mathrm{H}_{0} \mathrm{~S}=2 \mathrm{KV}$, i.e., for $h_{0}=1$.

The parameter $h_{s p}$ contains information about the spinphonon coupling. The spin-phonon coupling energy is written as $\varepsilon_{s p}=\sqrt{\pi \hbar^{3} \rho v^{5} / 3 \Gamma^{2}}$, where $\rho_{\mathrm{P}}$ is the nanoparticle density, $v$ is the sound velocity, and $\Gamma$ is the spin-phonon coupling strength. Actually, $\Gamma$ is a constant describing the intensity of the coupling between the magnetic nanoparticles and the phonon bath. The value of $\Gamma$ has been determined in Ref. 71 for Mn-ferrite nanoparticles. The value obtained by the authors along with the nanoparticle parameters corresponds to $h_{s p} \sim 10^{-2}$.

\section{REFERENCES}

'M. Khairul, E. Doroodchi, R. Azizian, and B. Moghtaderi, Energy Convers. Manage. 149, 660 (2017).

${ }^{2}$ E. E. Mazon, E. Villa-Martanez, A. Hernandez-Sámano, T. Cardova-Fraga, J. J. Ibarra-Sánchez, H. A. Calleja, J. A. L. Cruz, A. Barrera, J. C. Estrada, J. A. Paz, L. H. Quintero, and M. E. Cano, Rev. Sci. Instrum. 88, 084705 (2017). ${ }^{3}$ B. B. Lahiri, S. Ranoo, and J. Philip, J. Phys. D Appl. Phys. 50, 455005 (2017).

${ }^{4}$ M. A. Zayed, M. A. Ahmed, N. G. Imam, and D. H. El Sherbiny, J. Supercond. Novel Magn. 29, 2899 (2016).

${ }^{5}$ B. Mehdaoui, R. P. Tan, A. Meffre, J. Carrey, S. Lachaize, B. Chaudret, and M. Respaud, Phys. Rev. B 87, 174419 (2013).

${ }^{6}$ O. O. Ahsen, U. Yilmaz, M. D. Aksoy, G. Ertas, and E. Atalar, J. Magn. Magn. Mater. 322, 3053 (2010).

${ }^{7}$ P. Cantillon-Murphy, L. Wald, E. Adalsteinsson, and M. Zahn, J. Magn. Magn. Mater. 322, 727 (2010).

${ }^{8}$ C.-H. Fang, P.-I. Tsai, S.-W. Huang, J.-S. Sun, J. Z.-C. Chang, H.-H. Shen, S.-Y. Chen, F. H. Lin, L.-T. Hsu, and Y.-C. Chen, BMC Infect. Dis. 17, 516 (2017).

${ }^{9}$ C. S. B. Dias, T. D. M. Hanchuk, H. Wender, W. T. Shigeyosi, J. Kobarg, A. L. Rossi, M. N. Tanaka, M. B. Cardoso, and F. Garcia, Sci. Rep. 7, 14843 (2017).

${ }^{10}$ A. Lunghi, F. Totti, R. Sessoli, and S. Sanvito, Nat. Commun. 8, 14620EP (2017).

${ }^{1}$ S. Mornet, S. Vasseur, F. Grasset, and E. Duguet, J. Mater. Chem. 14, 2161 (2004).

${ }^{12}$ F. F. Fachini and A. F. Bakuzis, J. Appl. Phys. 108, 084309 (2010).

${ }^{13}$ C. F. C. Cristaldo and F. F. Fachini, Phys. Fluids 25, 037101 (2013).

${ }^{14}$ C. F. Cristaldo and F. F. Fachini, Combust. Flame 160, 1458 (2013).

${ }^{15}$ E. L. Jr, E. D. Biasi, M. V. Mansilla, M. E. Saleta, M. Granada, H. E. Troiani, F. B. Effenberger, L. M. Rossi, H. R. Rechenberg, and R. D. Zysler, J. Phys. D Appl. Phys. 46, 045002 (2013).
${ }^{16}$ E. Sadeghinezhad, M. Mehrali, A. R. Akhiani, S. T. Latibari, A. Dolatshahi-Pirouz, H. S. C. Metselaar, and M. Mehrali, Appl. Therm. Eng. 114, 415 (2017).

${ }^{17}$ F. Fadaei, M. Shahrokhi, A. M. Dehkordi, and Z. Abbasi, J. Magn. Magn. Mater. 429, 314 (2017).

${ }^{18}$ E. D. Vtulkina and E. A. Elfimova, J. Magn. Magn. Mater. 431, 218 (2017).

${ }^{19}$ K. D. Usadel, Phys. Rev. B 95, 104430 (2017).

${ }^{20}$ S. A. Shah, D. B. Reeves, R. M. Ferguson, J. B. Weaver, and K. M. Krishnan, Phys. Rev. B 92, 094438 (2015).

${ }^{21}$ S. H. Dubina and L. E. Wedgewood, Phys. Fluids 29, 092001 (2017).

${ }^{22}$ P. de la Presa, Y. Luengo, M. Multigner, R. Costo, M. P. Morales, G. Rivero, and A. Hernando, J. Phys. Chem. C 116, 25602 (2012).

${ }^{23}$ M. I. Shliomis, Sov. Phys. Uspekhi 17, 153 (1974).

${ }^{24} \mathrm{M}$. I. Shliomis and K. I. Morozov, Phys. Fluids 6, 2855 (1994).

${ }^{25}$ R. E. Rosensweig, J. Magn. Magn. Mater. 252, 370 (2002).

${ }^{26}$ R. J. Tackett, J. Thakur, N. Mosher, E. Perkins-Harbin, R. E. Kumon, L. Wang, C. Rablau, and P. P. Vaishnava, J. Appl. Phys. 118, 064701 (2015).

${ }^{27}$ R. Regmi, A. Naik, J. S. Thakur, P. P. Vaishnava, and G. Lawes, J. Appl. Phys. 115, $17 \mathrm{~B} 301$ (2014).

${ }^{28}$ H.-D. Pfannes, A. Mijovilovich, R. Magalhães Paniago, and R. Paniago, Phys. Rev. B 62, 3372 (2000).

${ }^{29}$ J. Carrey, B. Mehdaoui, and M. Respaud, J. Appl. Phys. 109, 083921 (2011).

${ }^{30} \mathrm{~N}$. A. Usov, J. Appl. Phys. 107, 123909 (2010).

${ }^{31}$ R. Hergt, S. Dutz, and M. Raider, J. Phys. Condens. Matter 20, 385214 (2008).

${ }^{32}$ A. Lefebvre, Gas Turbine Combustion (Taylor \& Francis, 1983).

${ }^{33} \mathrm{~F}$. Williams, Combustion Theory (The Benjamin/Cummings Publishing Company, Inc., 1985).

${ }^{34}$ K. Kuo, Principles of Combustion (John Wiley \& Sons, 1986).

${ }^{35} \mathrm{~J}$. Ramos, Internal Combustion Engine Modeling (Hemisphere Publishing Corporation, 1989)

${ }^{36} \mathrm{~W}$. Sirignano, Fluid Dynamics and Transport of Droplets and Sprays (Cambridge University Press, 2000).

${ }^{37} \mathrm{C}$. Law, Combustion Physics (Cambridge University Press, 2006).

${ }^{38} \mathrm{~J}$. Villain, F. Hartman-Boutron, R. Sessoli, and A. Rettori, Europhys. Lett. 27, 159 (1994).

${ }^{39}$ P. Khuntia, S. Manni, F. R. Foronda, T. Lancaster, S. J. Blundell, P. Gegenwart, and M. Baenitz, Phys. Rev. B 96, 094432 (2017).

${ }^{40} \mathrm{Q}$. L. Vuong, P. Gillis, A. Roch, and Y. Gossuin, Wiley Interdiscip. Rev. Nanomed. Nanobiotechnol. 9, 299-310 (2017).

${ }^{41}$ V. I. Bakhmutov and A. Clearfield, J. Phys. Chem. C 121, 7372 (2017).

${ }^{42} \mathrm{~J}$. Li, R. Fan, H. Wang, B. Ye, B. Zeng, H. Zhai, X. Peng, and J. Du, Phys. Rev. X 7, 031011 (2017).

${ }^{43}$ D. Gatteschi, R. Sessoli, and J. Villain, Molecular Nanomagnets, Mesoscopic Physics and Nanotechnology (OUP, Oxford, 2011).

${ }^{44}$ C. Sangregorio, T. Ohm, C. Paulsen, R. Sessoli, and D. Gatteschi, Phys. Rev. Lett. 78, 4645 (1997).

${ }^{45}$ F. Luis, J. Bartolomé, and J. F. Fernández, Phys. Rev. B 57, 505 (1998).

${ }^{46}$ Y. Tian, S. Shen, J. Cong, L. Yan, S. Wang, and Y. Sun, J. Am. Chem. Soc. 138, 782 (2016).

${ }^{47} \mathrm{M}$. Yaari and A. Keren, Phys. Rev. B 95, 174435 (2017).

${ }^{48}$ M. D. Jenkins, Y. Duan, B. Diosdado, J. J. García-Ripoll, A. Gaita-Ariño, C. Giménez-Saiz, P. J. Alonso, E. Coronado, and F. Luis, Phys. Rev. B 95, 064423 (2017).

${ }^{49} \mathrm{E}$. Merzbacher, Quantum Mechanics (Wiley, 1998).

${ }^{50}$ W. F. Brown, Phys. Rev. 58, 736 (1940).

${ }^{\mathbf{5 1}}$ W. F. Brown, Phys. Rev. 130, 1677 (1963).

${ }^{\mathbf{5 2}}$ Y. P. Kalmykov, S. V. Titov, and W. T. Coffey, Phys. Rev. B 86, 104435 (2012).

${ }^{53}$ C. N. Marin, I. Malaescu, and P. C. Fannin, J. Therm. Anal. Calorim. 119, 1199 (2015).

${ }^{54}$ S.-J. Park, S. Kim, S. Lee, Z. G. Khim, K. Char, and T. Hyeon, J. Am. Chem. Soc. 122, 8581 (2000).

${ }^{55}$ L. M. Parkes, R. Hodgson, L. T. Lu, L. D. Tung, I. Robinson, D. G. Fernig, and N. T. K. Thanh, Contrast Media Mol. Imaging 3, 150 (2008). 
${ }^{\mathbf{5 6}}$ G. G. Couto, J. J. Klein, W. H. Schreiner, D. H. Mosca, A. J. de Oliveira, and A. J. Zarbin, J. Colloid Interface Sci. 311, 461 (2007).

${ }^{57}$ A. G. Kolhatkar, A. C. Jamison, D. Litvinov, R. C. Willson, and T. R. Lee, Int. J. Mol. Sci. 14, 15977 (2013).

${ }^{58}$ J.-R. Jeong, S.-J. Lee, J.-D. Kim, and S.-C. Shin, Phys. Status Solidi B 241, 1593 (2004).

${ }^{\mathbf{5 9}}$ S. Maenosono and S. Saita, IEEE Trans. Magn. 42, 1638 (2006).

${ }^{60} \mathrm{~F}$. Cverna and A. Committee, ASM Ready Reference: Thermal Properties of Metals, ASM Ready Reference Series (ASM International, 2002).

${ }^{61}$ R. Kappiyoor, M. Liangruksa, R. Ganguly, and I. K. Puri, J. Appl. Phys. 108, 094702 (2010).

62 J. Emsley, The Elements, Oxford Chemistry Guides (Clarendon Press, 1998).

${ }^{63}$ S. Blundell, Magnetism in Condensed Matter, Oxford Master Series in Condensed Matter Physics (OUP, Oxford, 2001).
${ }^{64}$ R. Grau-Crespo, A. Y. Al-Baitai, I. Saadoune, and N. H. D. Leeuw, J. Phys. Condens. Matter 22, 255401 (2010).

${ }^{65}$ M. S. Seehra, V. Singh, P. Dutta, S. Neeleshwar, Y. Y. Chen, C. L. Chen, S. W. Chou, and C. C. Chen, J. Phys. D Appl. Phys. 43, 145002 (2010).

${ }^{66} \mathrm{C}$. Kittel, Introduction to Solid State Physics (Wiley, 1996).

${ }^{67} \mathrm{H}$. El Ghandoor, H. Zidan, M. M. Khalil, and M. Ismail, Int. J. Electrochem. Sci. 7, 5734 (2012).

${ }^{68}$ I. Malaescu, C. N. Marin, M. Bunoiu, P. C. Fanin, N. Stefu, and L. Iordaconiu, Ann. West Univ. Timisoara Phys. 58, 81 (2014).

${ }^{69} \mathrm{~J}$. Heywood, Internal Combustion Engine Fundamentals (McGraw-Hill Education, 1988).

${ }^{70}$ F. R. S. E. C. Stoner and E. P. Wohlfarth, Philos. Trans. R. Soc. Lond. A 240, 599 (1948).

${ }^{71}$ H.-D. Pfannes, J. Dias Filho, J. López, S. Pereira, P. Morais, and F. Tourinho, Hyperfine Interact. 113, 507 (1998). 